\title{
Design and Implementation of RF Deployed SMS based Equipment Controller
}

\author{
Sadeque Reza Khan \\ Lecturer, Dept. of EEE \\ Prime University \\ Dhaka, Bangladesh
}

\author{
Ahmed Al Mansur \\ Lecturer, Dept. of EEE \\ Prime University \\ Dhaka, Bangladesh
}

\author{
Muhammad Towhidur Rahman \\ Lecturer, Dept. of EEE \\ The University of Asia Pacific \\ Dhaka, Bangladesh
}

\begin{abstract}
Short messaging Service (SMS) is popularly used to provide information access to people on the move. This has resulted in advances in mobile communication bring great effects on people's life styles. This paper describes the implementation of a remote control scheme, remote control system via SMS, which controls home and factory devices in short distance and long distance, respectively. This paper proposes a SMS-based protocol, which is designed with the mechanisms of reliable transmission and information encryption, thus it is capable of the implementation of secure and reliable control. This paper mainly focuses on the controlling of home and factory appliances remotely. The system is SMS based and uses wireless technology to revolutionize the standards of living. As the system is wireless therefore more adaptable and costeffective. The whole system is controlled by a PIC microcontroller. We can control a good number of systems or devices by this method.
\end{abstract}

\section{General Terms}

Modem, Micro-controller, Opto-coupler.

\section{Keywords}

Maxx232, RS232, Soft touch switch, SMS, Wireless Sensor.

\section{INTRODUCTION}

Early in 1930s, the perspective of "Machine for Living" was proposed; in 1950s, the term "Automated Home" emerged; nowadays, terms such as "Digital Home", "Intelligent Home" and "Smart Home" come forth [1]. Smart Home integrates technologies such as computer, network, and automata to provide people an effective, comfortable, convenient and secure living environment. SMS, which transmits messages through Global System for Mobile Communication (GSM) networks, is a very popular service. The access to GSM networks is easier than to Internet, so the control system for home and factory with mobile phone via SMS can be applied in a wider area than webpage [2], [3]. Modern Industrial control systems tend to adopt a variety of intelligent wireless device controllers to interface front-end computers with active components of the industrial device. Some of the intelligent control systems come equipped with a wireless SMS interface as well.

The aim of this paper is to provide a simple system which is SMS-based and controls our home and industrial equipments with a Radio Frequency (RF) controller without any complexity. The heart of this system is PIC microcontroller. This system is divided in two parts; one is used to process the received SMS and send it via RF module and second part is used to control the devices.

\section{WORK OVERVIEW}

The design steps and working principles of the system is organized into different units, one is Information receive Process and Transmit (IPT) unit and second is Device controller (DC) unit. IPT unit is the part of system that is actually used to receive the SMS convert it into data packet form and transmit it to the DC unit. DC unit's job is to collect data packet, convert it into parallel data and on the switch of a corresponding device by using a soft touch switch. Figure 1 shows the design of IPT unit and DC unit using block diagram.

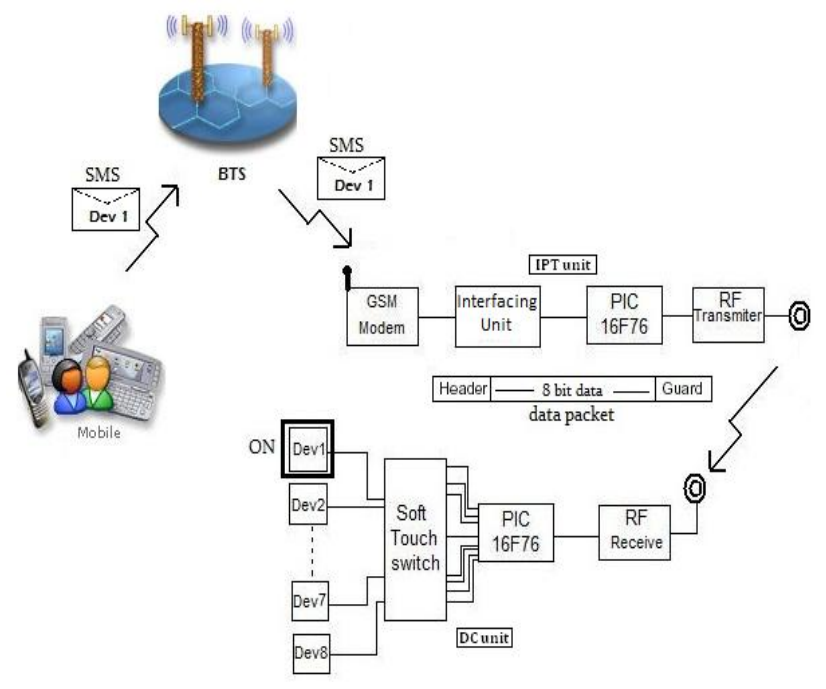

Fig 1: Design of IPT unit and DC unit

\subsection{System Design: IPT Unit}

\subsubsection{SMS}

SMS is defined as a text-based service that enables up to 160 characters to be sent from one mobile phone to another. In a similar vein to email, messages are stored and forwarded at an SMS centre, allowing messages to be retrieved later if you are not immediately available to receive them [4]. Unlike voice calls, SMS messages travel over the mobile network's lowspeed control channel. Using the convenience of SMS, this project lets you remotely control equipment by sending plain text messages, such as "pump on", "aircon off", and "reset" or "light1 on" - all of which can be pre-programmed into the controller and easily remembered later. It can control up to eight external devices. The 8 bit microcontroller is used to control the whole circuit. It read message from the RF modem and control the devices according the received message. 


\subsubsection{Modem}

Modem stands for "modulator / demodulator" and it encodes and decodes signals sent to and from the network servers. A wireless modem shown in fig 2 is a network device which connects to a wireless network [5]. Modems are frequently associated with telephone systems, but wireless modems are used with computers and also with communication mediums. Wireless modem interfaces include PCMCIA, Compact Flash, USB and Serial Port. In this project we interface the modem through a Serial Port with a microcontroller IC.

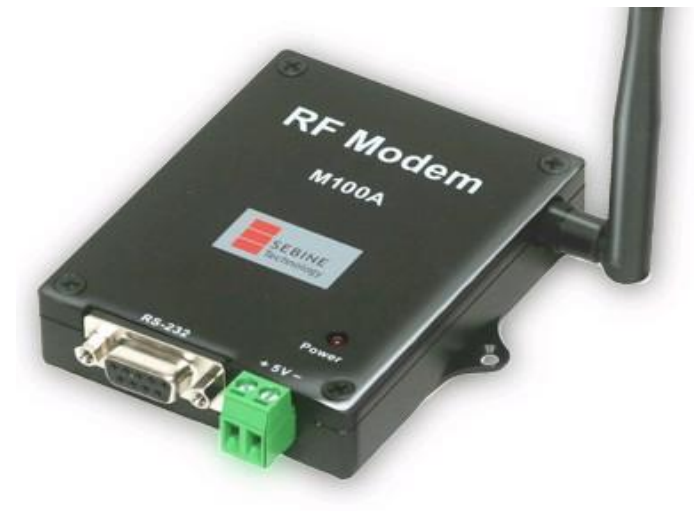

Fig 2: Wireless modem

\subsubsection{Interface Unit}

Max-232: The MAX232 is a dual driver/receiver that includes a capacitive voltage generator to supply EIA-232 voltage levels from a single 5-V supply. Each receiver converts EIA232 inputs to 5-V TTL/CMOS levels [6]. These receivers have a typical threshold of $1.3 \mathrm{~V}$ and a typical hysteresis of $0.5 \mathrm{~V}$, and can accept $30-\mathrm{V}$ inputs. Each driver converts TTL/CMOS input levels into EIA-232 levels. The driver, receiver, and voltage-generator functions are available as cells in the Texas Instruments in ASIC library.

RS232: RS232 is the most known serial port used in transmitting the data in communication and interface [7]. Even though serial port is harder to program than the parallel port, this is the most effective method in which the data transmission requires less wires that yields to the less cost. The RS232 is the communication line which enables the data transmission by only using three wire links. Db9 serial port has output voltage of $+12 \sim-12 \mathrm{~V}$ or $+25 \sim-25 \mathrm{~V}$. It can approximately transmit data up to 10 meter distance without any trouble. We can transmit data through serial port by using only three wires.

\subsubsection{RF Transmitter and Receiver}

Remote Unit uses RF wireless modules to communicate with Base Station using $2.4 \mathrm{Kbps}$ bit rate and high frequency of 433.92MHz using ASK modulation technique. RLP/TLP 434 RF Modules has been used for this purpose with the maximum power rating of $10 \mathrm{~dB}$ [8]. These RF modules can communicate with maximum baud rate of $200 \mathrm{Kbps}$ and can operate within a voltage range of 3.3-6 volts and the typical operating current is only $4.5 \mathrm{~mA}$. This module works directly with HT12D or similar decoder like microcontroller IC. Its Dimensions is of width - $43.4 \mathrm{~mm}$ and height $-11.5 \mathrm{~mm}$ (Excluding Pins). The transmitter (TLP434A) unit uses serial data coming from the microcontroller and Transmit that data using ASK modulation technique while the receiver (RLP434A) will receive the data and send it to the microcontroller serially. Figure 3 shows the RLP 434a receiver and TLP 434a transmitter.

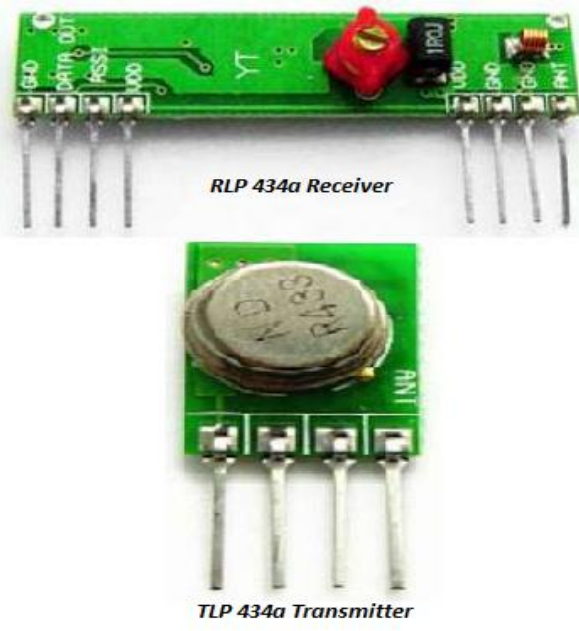

Fig 3: RLP/TLP 434a RF Modules

\subsubsection{Controller Unit}

The control module is built with the microcontroller IC. The central controller is Microchip PIC16F76. Microcontroller is the one of the ways of the evolution of microprocessor. It consists of a microprocessor, RAM, EEPROM or EPROM, I/O capacities, ADC, timer, interrupt controller and embedded controller. The microcontroller chip has the versatility to sense inputs and control outputs in the devices. F-family has been selected because it can be burned 1 million times without enabling code protection option. PIC 16F76 is a mid range and 16 series low cost 8 bit microcontroller [9]. It consists of $28 \mathrm{I} / \mathrm{O}$ (Bi directional lines) with $25 \mathrm{~mA}$ current in per pin. It also has five channel built-in A/D converter and serial communication.

\subsubsection{Working Diagram of IPT Unit}

The schematic diagram of Information receives Process and Transmit unit IPT Unit is shown in fig 4. Using a flowchart the total process of the IPT Unit is shown in fig 5.

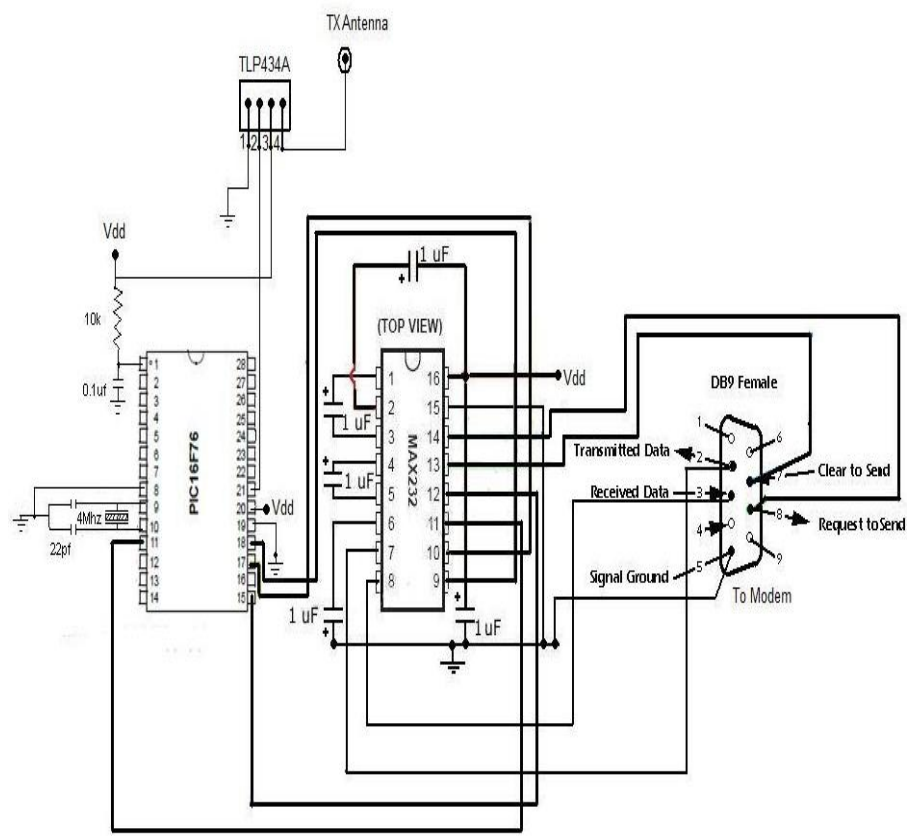

Fig 4: Schematic Diagram of IPT Unit 


\subsection{Device Controller (DC) Unit}

\subsubsection{RF transmitter and Receiver}

A RLP/TLP 434 RF module similar to that used in IPT has been employed in DC unit. The receiver is always online since it is always waiting for incoming data but transmitter will be turned on whenever a SMS is in progress.

\subsubsection{Control Module}

The central control system is designed using PIC16F76 as it has a built-in USART (Universal Serial Asynchronous Receiver Transmitter) and serial to parallel conversion capability.

\subsubsection{PIC microcontroller $16 f 72$}

PIC $16 \mathrm{~F} 72$ is a mid range and 16 series low cost 8 bit microcontroller [8]. It consists of $28 \mathrm{I} / \mathrm{O}$ (Bi directional lines) with $25 \mathrm{~mA}$ current in per pin. It also has five channel built-in $\mathrm{A} / \mathrm{D}$ converters with 8 levels deep hardware stacks.

\subsubsection{Optocoupler}

The 4N35 is an optocoupler for general purpose applications. It contains a light emitting diode optically coupled to a phototransistor. It can give up to $3550 \mathrm{Vrms}$ isolation [10].

\subsubsection{Relay}

A relay is an electrical switch that opens and closes under the control of another electrical circuit. In the original form, the switch is operated by an electromagnet to open or close one or many sets of contacts.

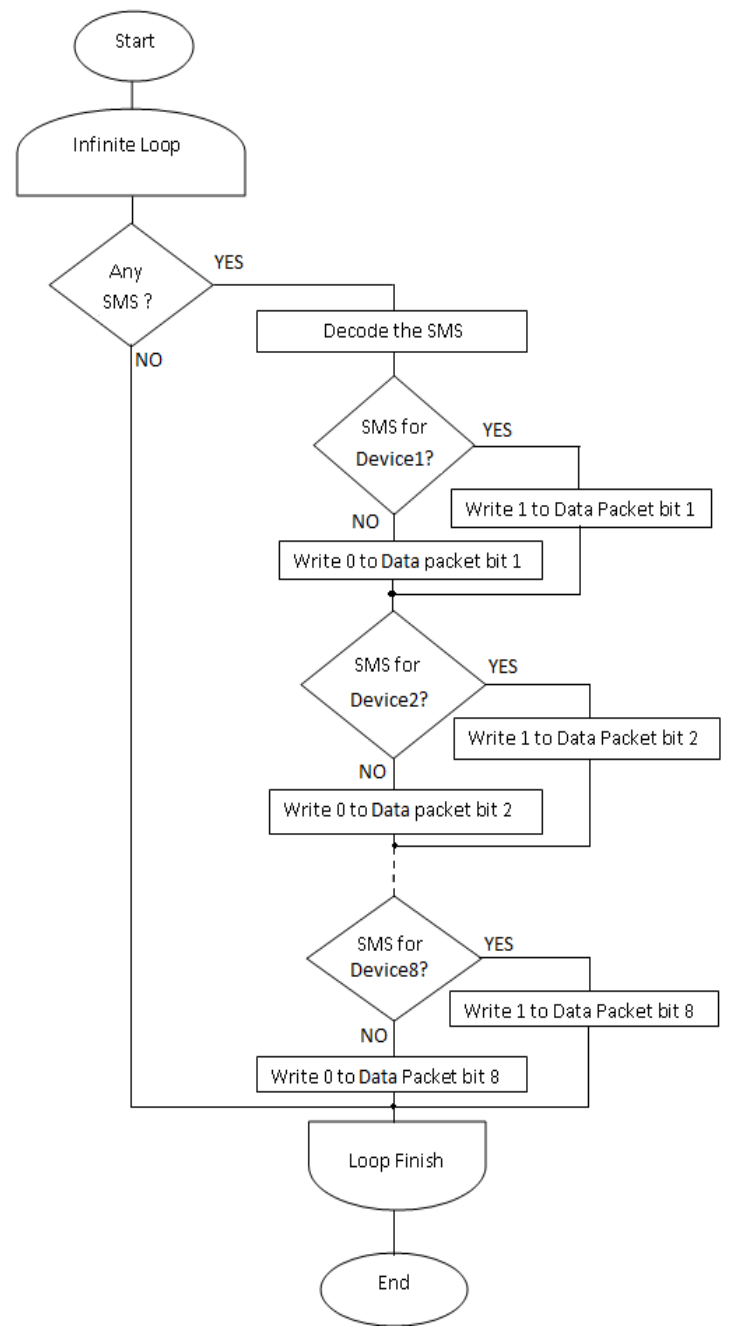

Fig 5: Flowchart of IPT Unit

\subsubsection{Soft touch switch}

The switch which will make a device on in one push and off in the next push and which operates in $5 \mathrm{~V}$ DC but can be used to drive $220 \mathrm{~V} \mathrm{AC}$; that is called a soft touch switch. Figure 6 shows the schematic diagram of soft touch switch unit.

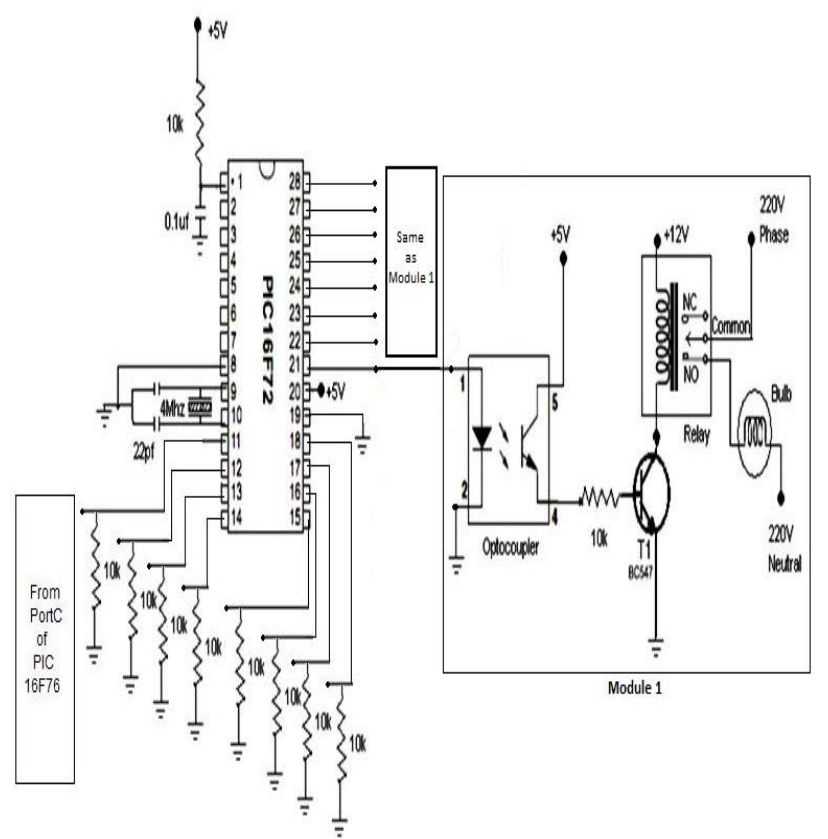

Fig 6: Schematic Diagram of Soft touch switch unit

\subsubsection{Working Diagram of DC Unit}

The schematic diagram of Device Controller Unit is shown in fig 4. The control process of the DC Unit is shown in fig 5 using a flowchart.

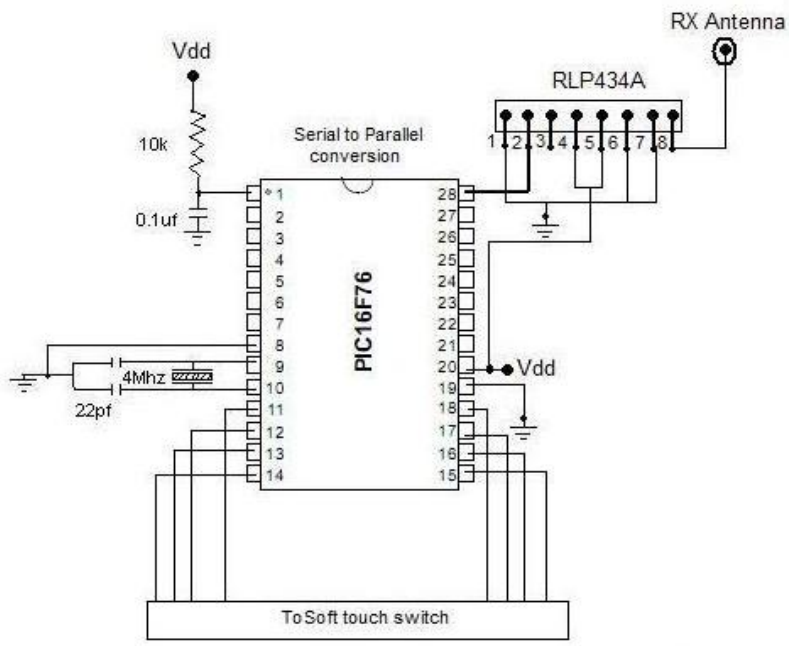

Fig 7: Schematic Diagram of DC Unit 


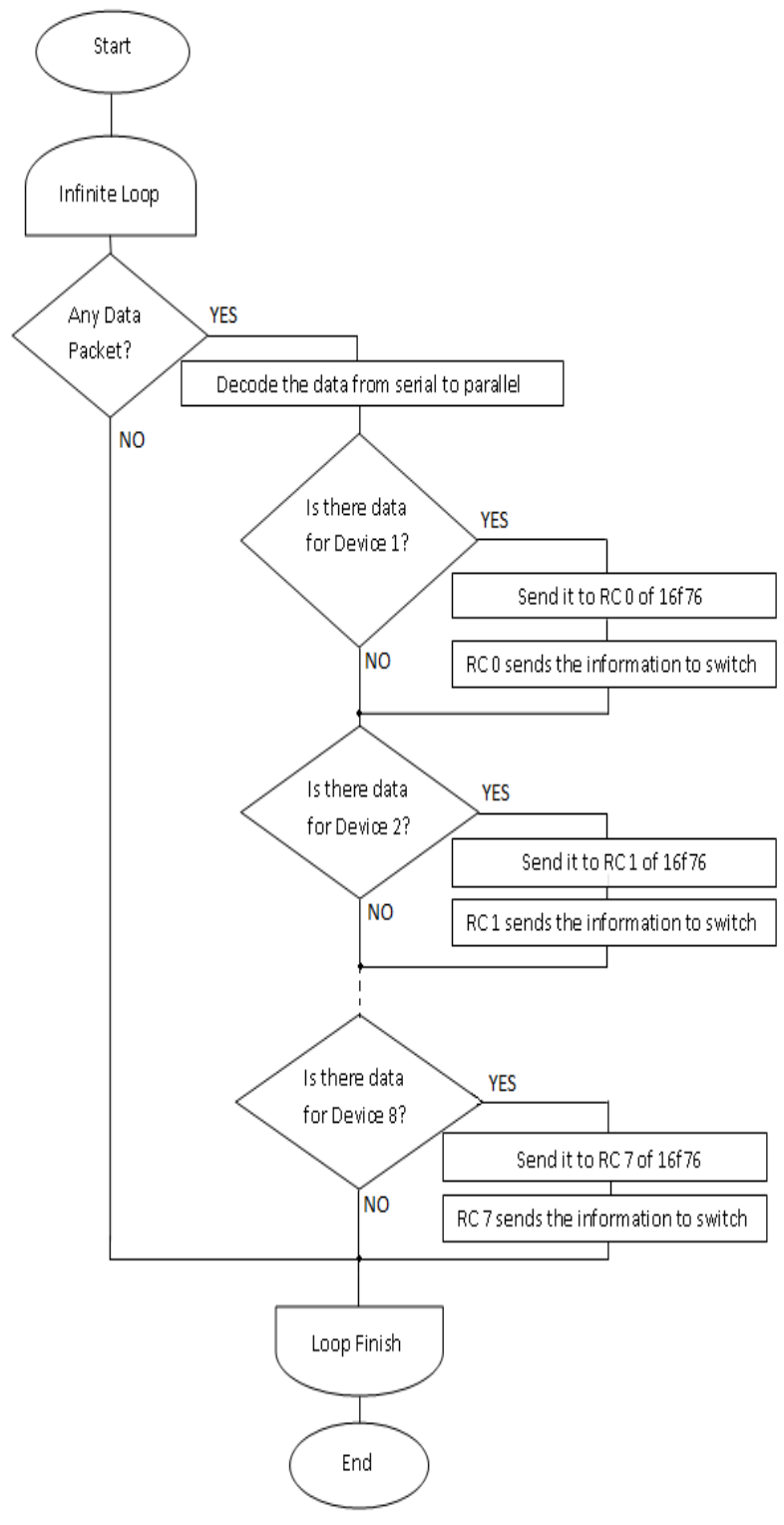

Fig 8: Flowchart of DC Unit

\section{CONCLUSION}

The aim of this project is to make our home and factory devices more controlled efficient to use. So times it is required for us to control a device of an industry from a remote distance than this project can make the work easier. As GSM network is now available mostly in all areas so this system will be very helpful for a person who want to control his own devices. Though SMS based different systems are available our proposed system is less complex, easy to install, cost effective and easy to operate as well.

\section{REFERENCES}

[1] YUAN Xiao-Chen, CHEANG Chak-Fong and LI JianQing, "Implementation of a Remote Control System for Smart Home", 2007, pp: 44-49.

[2] X. Y. Zhou and D. Shi, "Development and Application of SMS and WAP", Beijing, Publishing House of Electronics Industry Press, 2002-12.

[3] Guthery, S. B., Cronin, M. J., Tian, M. and Huang, Y., "Mobile Application Development with SMS and the SIM Toolkit", Beijing, Post and Telecom Press, 2003-9.

[4] SMS Gateway \& Application Software, 2011 [Online]. Available:http://www.logixmobile.com/products/swiftsm $\mathrm{s} /$ index.asp

[5] Wireless modem, December 2011 [Online]. Available: http://en.wikipedia.org/wiki/Wireless modem.

[6] MAX232 Serial level converter August 2010 [Online]. Available: http://www.SoDoItYourself.com./MAX232.

[7] Serial communication via RS-232, 28th July 2010 [Online]. Available: http://www.electrosofts.com /bioscom example.

[8] RF Transmitter and Receiver, 30th April 2010 [Online]. Available:http://www.sparkfun.com/datasheets/RF/KLP_ walkthrough.pdf

[9] PIC 16F76, December 2011 [Online]. Available: http://www.microchip.com/wwwproducts/Devices.aspx

[10] AGILENT 4N35 phototransistor optocoupler, July 2010 Available:www.datasheetcatalog.com. 